\title{
A human neuronal model of Niemann Pick $C$ disease developed from stem cells isolated from patient's skin
}

Natascha Bergamin ${ }^{1 \dagger}$, Andrea Dardis ${ }^{2 \dagger}$, Antonio Beltrami ${ }^{1}$, Daniela Cesselli ${ }^{1}$, Silvia Rigo ${ }^{1}$, Stefania Zampieri ${ }^{2}$, Rossana Domenis ${ }^{1}$, Bruno Bembi ${ }^{2}$ and Carlo Alberto Beltrami ${ }^{1^{*}}$

\begin{abstract}
Background: Niemann Pick C (NPC) disease is a neurovisceral lysosomal storage disorder due to mutations in NPC1 or NPC2 genes, characterized by the accumulation of endocytosed unesterified cholesterol, gangliosides and other lipids within the lysosomes/late endosomes. Even if the neurodegeneration is the main feature of the disease, the analysis of the molecular pathways linking the lipid accumulation and cellular damage in the brain has been challenging due to the limited availability of human neuronal models.

Objective: The aim of this study was to develop a human neuronal model of NPC disease by inducing neuronal differentiation of multipotent adult stem cells (MASC) isolated from NPC patients.

Methods: Stem cells were isolated from 3 NPC patients and 3 controls both from skin biopsies and previously established skin fibroblast cultures. Cells were induced to differentiate along a neuronal fate adapting methods previously described by Beltrami et al, 2007. The surface immunophenotype of stem cells was analyzed by FACS. Stem cell and neuronal markers expression were evaluated by immunofluorescence. Intracellular accumulation of cholesterol and gangliosides were assessed by filipin staining and immunofluorescence, respectively. A morphometric analysis was performed using a Neurite outgrowth image program.

Results: After 3 passages in selective medium, MASC isolated either from skin biopsies or previously established skin fibroblast cultures displayed an antigenic pattern characteristic of mesenchymal stem cells and expressed the stem cell markers Oct-4, Nanog, Sox-2 and nestin. A massive lysosomal accumulation of cholesterol was observed only in cells isolated from NPC patients. After the induction of neural differentiation, remarkable morphologic changes were observed and cells became positive to markers of the neuronal lineage NeuN and MAP2.

Differentiated cells from NPC patients displayed characteristic features of NPC disease, they showed intracellular accumulation of unesterified cholesterol and GM2 ganglioside and presented morphological differences with respect to cells derived from healthy donors.

In conclusion, we generated a human neuronal model of NPC disease through the induction of differentiation of stem cells obtained from patient's easily accessible sources. The strategy described here may be applied to easily generate human neuronal models of other neurodegenerative diseases.
\end{abstract}

Keywords: Niemann Pick C, Human neuronal model, Adult stem cells, Neuronal differentiation

\footnotetext{
* Correspondence: beltrami @uniud.it

†Equal contributors

'Department of Medical and Biological Sciences (DSMB), University of Udine,

P.le Kolbe 3, 33100, Udine, Italy

Full list of author information is available at the end of the article
} 


\section{Introduction}

Niemann Pick C [NPC-MIM 257220; MIM607625] disease is a neurodegenerative lysosomal storage disorder due to mutations in NPC1 or NPC2 genes, characterized by the accumulation of endocytosed unesterified cholesterol, gangliosides and other lipids within the lysosomes/late endosomes. Both proteins are involved in the intracellular trafficking of cholesterol and other lipids. Thus, the deficiency of either of them leads to the accumulation of the endocytosed unesterified cholesterol, gangliosides and other lipids within the lysosome/late endosome compartment [1].

Clinically, NPC disease presents a highly variable phenotype ranging from fetal to adult age. Even though initial manifestations are typically systemic, including liver and spleen enlargement, the disease has been classified according to the age at onset of neurological symptoms in: severe infantile form (onset before $2 \mathrm{y}$ of age), late infantile form (onset between 3-5 y of age), juvenile form (onset between 5 and $16 \mathrm{y}$ ) and adult form (onset at age $>16$ y) $[1,2]$.

Approximately $95 \%$ of NPC patients present mutations in NPC1 gene (MIM 607623; chr 18q11-q12) [3,4], which encodes a membrane glycoprotein of 1,278 amino acids containing 13 transmembrane domains and localized in late endosomes [5]. The other $5 \%$ of patients present mutations in NPC2 gene (MIM 601015; chr 14q24.3) [6] encoding a soluble 151 amino acid protein that is present in the lumen of lysosomes.

Despite the progress in characterizing the biochemical and genetic defects in NPC disease, the mechanisms underlying the pathophysiology of this disorder are not clear and the currently available therapeutic interventions are limited. In particular, the analysis of the molecular pathways linking the lipid accumulation and cellular damage in the brain has been challenging due to the limited availability of neuronal models.

Two mouse models of NPC disease have been described and used to study NPC pathogenesis, the BALB $\backslash \mathrm{C}$ NPC [7] and the Npc1 (nmf164) mouse [8]. The naturally occurring BALB $\backslash \mathrm{c}$ NPC mouse recapitulates the main features of human pathology [7]. However, while this model presents a very severe phenotype, most NPC patients present with a less severe form of the disease. This issue is particularly relevant when this model is used to test new potential therapies since the very acute nature of the $B A L B \backslash c$ NPC mouse model may mask the potential benefits of therapies that could be useful in a clinical setting in patients. Recently, a new mouse model of NPC disease, Npc1 (nmf164) carrying a c.3163A>G mutation that results in an aspartate to glycine change at position 1005 (D1005G), has been generated. This mutant mouse displays a slower development of the
NPC phenotype than the BALB $\backslash \mathrm{C}$ NPC mouse. Therefore, it may represent a good model for the late-onset, slower progressing forms of NPC disease [8]. However, it is worth noting that some characteristic features of NPC human neurons are not present in mice, suggesting important species differences between mice and human NPC neurons [9-11].

Many studies have been performed in peripheral cells in culture. However, the obtained results might not be extrapolated to neuronal cells since the pattern of accumulated lipids is quite different between peripheral and central tissues [12].

Recent advances in human stem cell biology and the optimization of protocols for in vitro differentiation of stem cells into different cell lineages have opened new possibilities for generating disease cellular models.

Pluripotent cells have the ability to form all the body's cell lineages, including germ cells and some extraembryonic cell types [13]. The analyisis of blastocyst chimerism and tetraploid aggregation followed by gestation are the most appropriate tests employed to assess mouse stem cell pluripotency [13]. Human stem cell pluripotency cannot be tested for their ability to generate fully functional germ cells in vivo without raising ethical concern. Consequently, a surrogate test for human stem cell pluripotency is teratoma formation upon administration to a receptive animal [13]. Interestingly, a number of reports have demonstrated that adult human tissues host widely multipotent cells. Although these latter are able to differentiate, both in vitro and in vivo [13,14], into mesodermal [15], ectodermal [16] and endodermal [17] derivatives, even at a clonal level [18], they do not form either teratomas in vivo or contribute to the germ cell lineages, thus not entirely fulfilling the criteria for pluripotency. This may be secondary to epigenetic differences between adult and embryonic stem cells [14].

Our group has described a highly reproducible method to isolate, from different adult human tissues (heart, liver and bone marrow), a population of multipotent stem cells (named Multipotent Adult Stem Cells or MASC), which express several pluripotent state specific transcription factors (i.e. OCT4, Nanog, Sox2, and Rex1), display high levels of telomerase activity and show all the characteristics of stem cells, such as clonogenicity and self renewal. Furthermore, they present a highly similar gene expression profile, irrespectively from the tissue of origin and they maintain the ability to differentiate along some derivatives of the 3 germ layers, including neuroectoderm [19].

The aim of this study was to develop a human neuronal model of NPC disease by inducing neuronal differentiation of stem cells isolated from skin biopsies or fibroblasts in culture obtained from NPC patients. 


\section{Methods}

\section{Human samples}

Specimens from 3 patients affected by NPC disease (two of them were siblings) and 3 normal controls have been used in this study. Samples from a patient affected by Sandhoff disease were used as positive control for ganglioside accumulation assays.

All 3 NPC patients presented the classical biochemical phenotype characterized by massive lysosomal/ late endosomes accumulation of unesterified cholesterol in cultured fibroblasts. The diagnosis was confirmed by sequencing both NPC1 and NPC2 genes. All patients had mutations in the NPC1 gene. The two affected siblings presented the c.3182T $>C$ (P.I1061T)/ c.3182T $>C$ (p.I1061T) genotype, while the third unrelated NPC patient presented the c. $2795+1 \mathrm{G}>\mathrm{C} /$ c.3493G>A (p.V1165M) genotype. This study was approved by the ethical committee of the University Hospital "S. Maria della Misericordia" and written consent was obtained from all subjects.

\section{Stem cell selection and culture}

Stem cell enriched cultures were obtained, both from skin biopsies and from already established skin fibroblast cultures at early passages (P1, P2, P3), adapting the methods previously described $[19,20]$.

Briefly, skin biopsies were minced and digested with $0.04 \%$ of collagenase type I (Sigma-Aldrich) for 15 minutes at $37^{\circ} \mathrm{C}$. Cell suspension was filtered through a $40 \mu \mathrm{m}$ nylon membrane (Dako) and $4 \times 10^{4}$ cells $/ \mathrm{mm}^{2}$ were plated onto fibronectin coated dishes $(10 \mu \mathrm{g} / 100 \mathrm{~mm}$ plate, from Sigma-Aldrich). In alternative, $2 \times 10^{6}$ cells directly isolated from human skin biopsies or $1 \times 10^{6}$ cells obtained from confluent primary skin fibroblast cultures, were seeded onto $100 \mathrm{~mm}$ plates coated with fibronectin and expanded at least for three passages in a selective media composed of 60\% Dulbecco's Modified Eagle medium (DMEM)/40\% MCDB-201 (Sigma-Aldrich) supplemented with $1 \mathrm{mg} / \mathrm{ml}$ Linoleic Acid-BSA (SigmaAldrich);10 $10^{-9} \mathrm{M}$ dexamethasone (Sigma-Aldrich); $10^{-4} \mathrm{M}$ Ascorbic acid-2 phosphate (Sigma-Aldrich); 1X Insulintransferrin-sodium selenite (Sigma-Aldrich); $2 \%$ fetal bovine serum (FBS), (STEMCELL Technologies), $10 \mathrm{ng} / \mathrm{ml}$ human PDGF-BB (Peprotech EC); 10 ng/ml human EGF (Peprotech EC). Medium was replaced every 4 days and cells were split when they reached $70 / 80 \%$ confluence.

\section{Single cell cloning}

Stem cells, at the third passage in culture (P3), obtained from healthy donors ( $\mathrm{n}=600$ cells) and NPC patients ( $n=600$ cells) were individually deposited directly into fibronectin-coated wells of 96-well plates (Falcon, BD-Biosciences, Italy) with an automated cell sorter (FACSAria, BD), and cultured in expansion medium supplemented with $10 \%$ FBS $[19,20]$. To determine sorting efficiency and to verify if any well was seeded with more than one cell, we utilized the Vybrant CFDA SE (CFSE) as a cell tracker (Molecular Probes, Invitrogen). Wells were examined twice a week in order to determine the fraction of cells able to give rise to proliferating clones.

\section{Multilineage differentiation}

Multilineage differentiation was evaluated as previously described [19,20].

Muscle cell- and endothelial cell- differentiation was achieved plating 0.5 to $1 \times 10^{4} / \mathrm{cm}^{2}$ cells in expansion medium containing 5\% FBS (Sigma-Aldrich), $10 \mathrm{ng} / \mathrm{mL}$ bFGF, $10 \mathrm{ng} / \mathrm{mL}$ VEGF, and $10 \mathrm{ng} / \mathrm{mL}$ IGF-1 (all from Peprotech EC), but not EGF. Cells were allowed to become confluent and cultured for up to 2 weeks with medium exchanges every 4 days.

Hepatocytic differentiation was induced growing cells for two weeks at high density $\left(2 \times 10^{4} / \mathrm{cm}^{2}\right)$ onto fibronectin coated coverslips in a medium containing $0.5 \%$ FBS, $10 \mathrm{ng} / \mathrm{ml} \mathrm{FGF-4} \mathrm{and} 20 \mathrm{ng} / \mathrm{ml} \mathrm{HGF}$ (both from Peprotech EC).

For neurogenic differentiation, stem cells obtained after 3 passages in selective medium, were seeded at a density of 8000 cells $/ \mathrm{cm}^{2}$ into 96 multiwell plates (BD Biosciences) or on coverslips. The differentiation protocol was adapted from a method previously described $[19,20]$. Briefly, cells were plated in medium containing DMEMHG with 10\% FBS (called N1 medium). After 24 hours the DMEM-HG was replaced with fresh medium supplemented with $1 \%$ of B27 (Invitrogen), $10 \mathrm{ng} / \mathrm{ml}$ EGF (Peprotech) and $20 \mathrm{ng} / \mathrm{ml}$ bFGF (Peprotech) (called $\mathrm{N} 2$ medium) for 5 days. Thereafter, cells were incubated for 24/48 hours in DMEM supplemented with $5 \mu \mathrm{g} / \mathrm{ml}$ insulin, $200 \mu \mathrm{M}$ of indomethacin and 0.5 mM IBMX (all from Sigma-Aldrich) without FBS (called N3 medium).

\section{Flow cytometry analysis}

After at least 3 passages in selective medium, stem cells were detached and stained with the following primary conjugated antibodies: CD10, CD13, CD29, CD49a, CD49b, CD49d, CD90, CD73, CD44, CD45, HLA-DR, CD117, CD34, CD271 (BD Biosciences), CD105, CD66e, KDR (Serotech), CD133 (Miltenyi Biotec), CXCR4, (R\&D), ABCG-2 (Chemicon International). The percentage of cells expressing all considered antigens was determined by flow cytometry analysis (CyAn, Beckman Coulter). Properly conjugated isotype matched antibodies were used as negative controls.

\section{Filipin staining}

Filipin staining was performed using the method described by Blanchette-Mackie et al. [21]. Briefly, cells grown on 
coverslips, were incubated in serum free medium for 24 hours and then treated for 24 hours with LDL enriched medium. Cells were rinsed with PBS and fixed with $3 \%$ paraformaldehyde. After washing them with PBS, the cells were incubated with $1.5 \mathrm{mg}$ of glycine $/ \mathrm{ml}$ PBS for 10 minutes, stained with filipin (0.05 mg/ml, in PBS 10\% FCS) for 2 hours and examined using a Zeiss fluorescence microscope.

\section{Periodic acid Schiff staining (PAS)}

PAS was employed to detect glycogen accumulation. Slides were oxidized in $1 \%$ periodic acid for 5 minutes, rinsed three times in distilled water and treated with Schiff's reagent for 25 minutes. After extensive washing, slides were stained with Mayer's hematoxylin for 10 minutes.

\section{Immunofluorescence microscopy}

Protein markers: Cells were grown on coverslips then fixed in $4 \%$ paraformaldehyde for 20 minutes at room temperature, permeabilized 10 minutes at room temperature with $0.1 \%$ Triton X-100 (Sigma-Aldrich) and stained overnight at $4{ }^{\circ} \mathrm{C}$ to visualize stem cell markers: Oct4 (Abcam, Rabbit polyclonal, 1:150), Nanog (Abcam, Rabbit polyclonal, 1:200), Sox2 (Millipore, Mouse monoclonal, 1:200), Nestin (Millipore, 1:100); myocytes specific markers: smooth muscle actin (SMA) (Sigma, 1:50 mouse monoclonal) and $\alpha$-sarcomeric actin (ASA) (Sigma, 1:100 mouse monoclonal); endothelial cell marker: CD31 (Dako, 1:50 mouse monoclonal); hepatic specific marker: cytokeratins 8,18 , and 19 (CK) (1:50 mouse monoclonal); or neural specific markers: tubulin beta 3 (COVANCE, 1:1000 mouse monoclonal), NeuN, (Millipore, 1:50 mouse monoclonal) and MAP2 (Millipore, 1:50 rabbit polyclonal). Secondary antibody staining was done with donkey antirabbit or donkey anti-mouse antibodies (Alexa-Fluor 555 or 488, Molecular Probes) at 1:600 dilutions. Images were obtained with a live cell imaging dedicated system consisting of a Leica DMI 6000B microscope connected to a Leica DFC350FX camera (Leica Microsystems); 10X (numerical aperture: 0.25), 40X oil immersion (numerical aperture: 1.25) and 63X oil immersion (numerical aperture: 1.40) objectives were employed.

GM2 and GM3 analysis: The analysis of GM2 and GM3 gangliosides was performed as previously described [22]. Cells were grown on coverslips then fixed in $4 \%$ paraformaldehyde for 45 minutes at room temperature. Cells were then incubated at room temperature for 1 hour in blocking buffer [PBS with $10 \%$ normal donkey serum (NDS) and $0.02 \%$ saponin (Sigma-Aldrich)] and stained overnight at $4{ }^{\circ} \mathrm{C}$ with mouse anti-GM2 (1:20 in blocking buffer) or mouse anti-GM3 (1:20 in blocking buffer). Cells were then incubated with a TRITC conjugated donkey anti-mouse IgM [1:80 in PBS with 2\% NDS and $0.02 \%$ saponin
(Sigma-Aldrich)]. For colocalization studies, a monoclonal anti-LIMP-1 (Novus Biologicals, Littleton, USA) was used as a primary antibody, and an Alexa fluorconjugated anti-mouse (Invitrogen, Carlsbad, CA, USA) as a secondary antibody.

In all cases, nuclei were stained by DAPI (Vector Laboratories, Inc) and Vectashield (Vector) was used as mounting medium. Epifluorescence and phase contrast images were obtained with a live cell imaging dedicated system consisting of a Leica DMI 6000B microscope connected to a Leica DFC350FX camera (Leica Microsystems); 10X (numerical aperture: 0.25), 40X oil immersion (numerical aperture: 1.25) and 63X oil immersion (numerical aperture: 1.40) objectives were employed. Adobe Photoshop software was utilized to compose and overlay the images, and adjust contrast (Adobe, USA). The counts of positive cells were done manually, considering, where possible, 100 positive events per sample.

\section{Real-time RT-PCR}

Total RNA was extracted from both non-confluent cultures of undifferentiated and differentiated cells at P3 using the TRIzol Reagent (Invitrogen). After treatment with DNase I (Ambion), first strand cDNA synthesis was performed with $1 \mu \mathrm{g}$ total RNA using random hexanucleotides and MMLV reverse transcriptase (Invitrogen). Primers were designed from available human sequences using the primer analysis software Primer3 (Additional file 1: Table S1). Quantitative RT-PCR was performed using Roche LightCycler 480 Real-Time PCR System and the LightCycler 480 SYBR Green I Master (Roche), following manufacturer's instructions. GAPDH was used as internal control for normalization. LightCycler 480 Basic software (Roche) utilized the second derivative maximum method to identify the crossing point $(\mathrm{Cp})$.

\section{Evaluation of apoptosis}

Apoptosis in differentiated cells at P3 was evaluated by staining of phosphatidylserine exposed on cell membranes with FITC labeled Annexin V, according to the manufacturer's instructions (Sigma-Aldrich) and analyzed by flow cytometry using a FACScan (Becton Dickinson, Franklin Lakes, NJ, USA).

\section{Morphological analysis}

Morphological analysis data were collected by using the BD Pathway bioimaging platform. Differentiated cells were first immunostained for the neural marker MAP-2, then images were acquired on a BD Pathway 855 using a 20X objective $(0.75 \mathrm{NA})$ in the form of $2 \times 2$ montage. The images were then analyzed using BD's Neurite Outgrowth Algorithm that automatically measure parameters describing neurite outgrowth. 


\section{Statistical analysis}

Statistical analysis was performed using Student's $t$ test or one-way ANOVA test, followed by Bonferroni posttest. The analyses were carried out using the software Prism, version 4.0c, GraphPad Software, San Diego, CA, USA; JMP7, SAS Institute Inc., Cary, NC, USA. $\mathrm{p}<0.05$ was considered statistically significant.

\section{Results}

\section{Stem cell characterization}

In order to isolate multipotent stem cells from NPC patient samples, we applied a protocol that was optimized for the growth of widely multipotent cells with mesenchymal features (named human multipotent adult stem cellshMASC) from several human tissues $[19,20]$ to both freshly obtained skin biopsies and previously established skin fibroblast cultures. If successful, this latter strategy would allow us to derive multipotent cells from already available bio-repositories of cell lines, obtained from patients suffering from rare diseases, such as NPC, for diagnostic purposes.

When exposed to a selective culture medium enabling the growth of hMASC, proliferating cell lines could be obtained from all skin biopsies. On the contrary, only those fibroblast cultures that had not been extensively expanded in vitro (< 3 passages in vitro) were responsive to these stringent culture conditions. After 3 passages in the selective medium, the cells acquired a homogeneous morphology as shown in Figure 1A-D. No differences were observed neither between cells obtained from NPC patients and normal controls nor between cells obtained from biopsies or from already established skin fibroblast cultures.

In order to characterize the selected cells, their surface immunophenotype was analyzed. As shown in Figure 1E and Table 1, cells obtained both from skin biopsies and fibroblast cell lines displayed an antigenic pattern characteristic of mesenchymal stem cells, very similar to the one observed in MASC isolated from heart, liver or bone marrow [19]. No major differences were observed between cells derived from NPC patients or normal controls.

Cultured cells were then evaluated for the expression of stem cell markers, such as the pluripotent state specific transcription factors Oct-4, Nanog and Sox- 2 and the intermediate filament nestin. As shown in Figure 1F-K, the vast majority of cells (65 to 90\%) expressed these markers and this expression was independent from the disease state. In fact, no significant differences were observed between NPC and normal cells or between cells isolated from both skin biopsies and early passages of fibroblast cell lines.

In order to investigate whether the pathologic phenotype was retained in these cells, the intracellular accumulation of unesterified cholesterol was analyzed by filipin staining. A massive accumulation of cholesterol within the endosomal/lysosomal compartment was observed in cells isolated from either skin biopsies or fibroblast cultures of NPC patients (Figure 1L-N).

Therefore, in the light of these data, we decided to further characterize stem cells derived from already established skin fibroblast cultures and use them to develop a neuronal differentiation model.

Cell cultures showed two of the major features of multipotent adult stem cells such as clonogenicity and wide differentiation capacity. Specifically, when sorted as single cells into the wells of 96-well Terasaki plates, skin-derived cells were able to form proliferating colonies within 2 weeks after seeding. It is worth noting that cells derived from NPC patients seem to be less clonogenic than cells derived from healthy controls. Even if the differences were not statistically significant, this result suggests that some stem cells features may be compromised in NPC disease. The cells were able to differentiate along derivatives of all the three germ layers (Figure 2, Additional file 2: Figure S1 and Additional file 3: Figure S2). In this regard, cultured cells generated not only neurectodermic derivatives (see below) but mesodermal and endodermal ones as well. Specifically, cells derived from both healthy controls and NPC patients cultured in a medium added with IGF-1, bFGF and VEGF expressed the myocyte specific markers alpha-sarcomeric actin (ASA) and smooth muscle actin (SMA) and the endothelial cell marker CD31 (Additional file 2: Figure S1). Additionally, cells exposed to hepatocyte differentiation medium assumed a globular shape and became positive for cytokeratins 8, 18, and 19 (CK). Moreover, they acquired some hepatocitic functions such as the ability to store glycogen as demonstrated by the PAS staining (Additional file 3: Figure S2).

Altogether these results indicate that cells obtained from fibroblast cultures of healthy controls and NPC patients exhibited almost an identical mesenchymal stem cell immunophenotype, expressed pluripotent state specific transcription factors, were clonogenic and multipotent; therefore, in analogy with our previous studies, we named them hSKIN-MASC.

\section{Neural differentiation}

Twenty-four hours after the induction of neural differentiation, hSKIN-MASC showed remarkable morphologic changes. As shown in Figure 2 (panels A-B) differentiated cells displayed an enlargement of the cellular bodies and the presence of long projections, closely resembling the morphology of neuronal cells. Importantly, the morphology of differentiated cells obtained from NPC patients and healthy donors was clearly distinct. In fact, with 

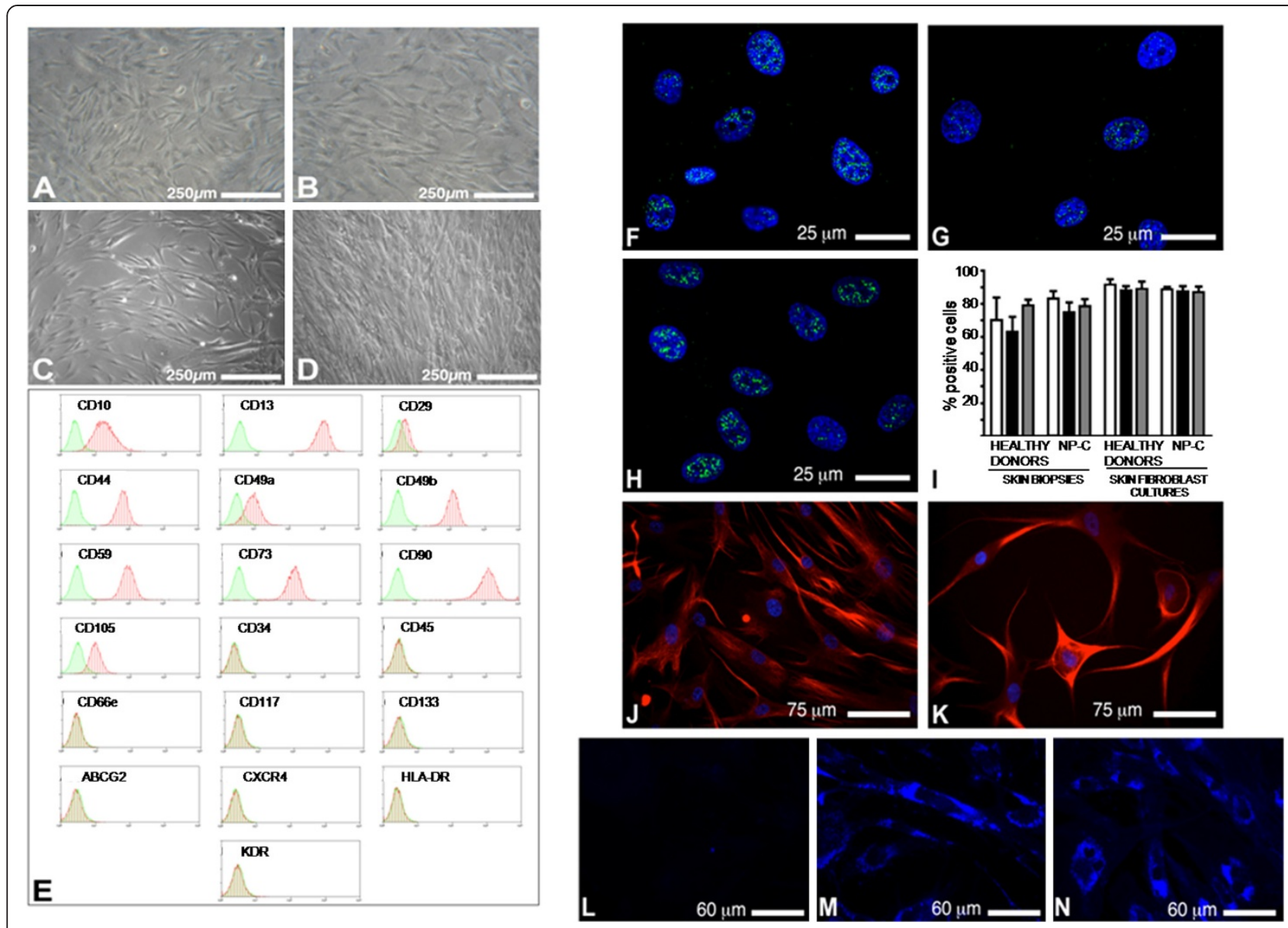

Figure 1 Characterization of stem cell populations obtained from skin biopsies or already established skin fibroblast cultures. (A-D) Phase contrast images of hSKIN-MASC at the third passage in culture: A-B) hSKIN-MASC obtained from skin biopsies of a healthy donor (A) and a NPC patient (B). C-D) hSKIN-MASC obtained from already established skin fibroblasts from healthy donor (C) and NPC patient (D). (E) Surface immunophenotype: representative flow cytometry histograms of skin derived stem cell cultures. Plots show isotype control lgG-staining profile (green histograms) versus specific antibody staining (red histograms). (F-H) Pluripotent state specific transcription factor expression: representative fluorescence images of Oct-4 (green fluorescence; $\mathbf{F}$ ), Nanog (green fluorescence; $\mathbf{G}$ ) and Sox-2 expression (green fluorescence; $\mathbf{H}$ ) localized in the nuclei of skin derived stem cell cultures. Nuclei are depicted by the blue fluorescence of DAPI staining (F-H). I) Quantification of the percentage of cells expressing Oct4 (white bars), Nanog (black bars) and Sox-2 (gray bars) in hSKIN-MASC obtained both from healthy donors ( $C T R L, n=3)$ and NPC $(n=3)$ skin biopsies or healthy donors (CTRL, $n=3)$ and NPC ( $n=3)$ skin fibroblast cultures. At least 400 cells have been counted for each cell line. Data are presented as mean \pm SD of 3 independent experiments. (J-K) Nestin expression (red fluorescence) in hSKIN-MASC obtained from a healthy donor (J) and a NPC patient (K). (L-N) Unesterified cholesterol accumulation: images obtained after performing the filipin staining (blue fluorescence) in hSKIN-MASC derived from a healthy donor skin biopsy (L), a NPC patient skin biopsy $(\mathbf{M})$ and a NPC skin fibroblast cell line $(\mathbf{N})$, respectively.

respect to healthy donors (Figure 2A), differentiated cells obtained from NPC patients (Figure 2B) were larger and presented numerous projections similar to dendrites.

Upon differentiation, a large fraction of hSKIN-MASC became positive to markers of the neuronal lineage. In particular, they expressed NeuN (Figure 2C-E), a neuronal specific nuclear protein, and MAP2 (Figure 2F-H), a structural protein specifically present in neuronal cells. Interestingly, the percentage of cells expressing NeuN was significantly lower in cells derived from NPC patients than in cells derived from healthy donors. In addition, differentiated cells did not express markers associated with glial differentiation, such as glial fibrillary acidic protein (GFAP) and oligodendroglial protein 4 (O4) (data not shown). These data suggest that the differentiation protocol described here specifically favored the differentiation towards the neuronal lineage. It is worth noting that, after differentiation, the cells became negative for the pluripotent state specific transcription factors Oct-4, Nanog and Sox-2, while the expression of nestin was maintained throughout the differentiation process.

To further characterize the type of neuronal cells obtained, mRNA expression of specific markers of dopaminergic (tyrosine hydroxylase, $\mathrm{TH}$ and dopamine transporter, DAT), cholinergic (choline acetyltransferase, CHAT) and GABAergic (glutamic acid decarboxylase, 
Table 1 Surface immunophenotype of stem cells isolated from skin biopsies or culture fibroblasts

\begin{tabular}{|c|c|c|c|c|}
\hline & Healthy donor (skin biopsy) & NP-C (skin biopsy) & Healthy donor (fibroblast culture) & NP-C (fibroblast culture) \\
\hline CD49d & $95.9 \pm 3.2$ & $99.89 \pm 0.22$ & $99.1 \pm 0.7$ & $99.9 \pm 0.08$ \\
\hline CD49a & $37.5 \pm 7.2$ & $48.6 \pm 0.4$ & $6.38 \pm 2.51$ & $54.18 \pm 3.44$ \\
\hline CD49b & $96.48 \pm 2.8$ & $99.21 \pm 0.3$ & $99.98 \pm 0.1$ & $99.95 \pm 0.2$ \\
\hline CD29 & $43.33 \pm 12.5$ & $62.75 \pm 1.05$ & $17.05 \pm 14.7$ & $17.62 \pm 4.9$ \\
\hline ABCG2 & $1.57 \pm 0.7$ & $1.14 \pm 0.02$ & $1.81 \pm 0.06$ & $1.37 \pm 0.25$ \\
\hline CXCR4 & $0.22 \pm 0.1$ & $0.16 \pm 0.01$ & $0.081 \pm 0.06$ & $0.18 \pm 0.05$ \\
\hline CD10 & $77.8 \pm 13$ & $98 \pm 0.4$ & $55.5 \pm 20.7$ & $96.05 \pm 1.2$ \\
\hline CD66 & $1.23 \pm 0.7$ & $3.04 \pm 0.05$ & $0.72 \pm 0.7$ & $0.28 \pm 0.23$ \\
\hline CD133 & $2.06 \pm 0.34$ & $5.88 \pm 0.28$ & $0.31 \pm 0.08$ & $0.23 \pm 0.12$ \\
\hline CD271 & $2.95 \pm 1.33$ & $0.2 \pm 0.071$ & $0.37 \pm 0.31$ & $0.89 \pm 0.24$ \\
\hline CD44 & $95.89 \pm 2.7$ & $97.25 \pm 0.2$ & $99.41 \pm 0.7$ & $98.94 \pm 1.02$ \\
\hline CD105 & $87 \pm 3.4$ & $85.9 \pm 2.78$ & $92.73 \pm 4.35$ & $74.92 \pm 30.8$ \\
\hline CD90 & $97.12 \pm 3.2$ & $98.88 \pm 1.41$ & $99.52 \pm 0.28$ & $96.25 \pm 3.7$ \\
\hline CD73 & $92.9 \pm 3.7$ & $99.75 \pm 0.08$ & $99.48 \pm 0.4$ & $99.7 \pm 0.19$ \\
\hline CD34 & $3.7 \pm 1.7$ & $1.9 \pm 0.1$ & $0.205 \pm 0.12$ & $0.66 \pm 0.83$ \\
\hline CD45 & $0.1 \pm 0.2$ & $0.19 \pm 0.01$ & $0.45 \pm 0.04$ & $0.07 \pm 0.02$ \\
\hline KDR & $3.12 \pm 1.1$ & $1.47 \pm 0.03$ & $1.92 \pm 2.18$ & $1.53 \pm 0.18$ \\
\hline CD13 & $98.49 \pm 3.7$ & $98.4 \pm 1.9$ & $99.78 \pm 0.21$ & $99.88 \pm 0.15$ \\
\hline CD117 & $1.1 \pm 0.67$ & $0.52 \pm 0.03$ & $0.15 \pm 0.13$ & $0.27 \pm 0.3$ \\
\hline HLA-DR & $0.37 \pm 0.1$ & $0.12 \pm 0.14$ & $0.65 \pm 1.01$ & $0.03 \pm 0.01$ \\
\hline
\end{tabular}

GAD) neurons were analyzed by real time PCR. None of these markers were detected in undifferentiated cells. After differentiation, both cells derived from healthy donors and NPC patients expressed similar levels of CHAT mRNA (Additional file 4: Figure S3), while the levels of TH, DAT and GAD mRNA were undetectable.

No differences in the levels of apoptosis have been detected between differentiated cells derived from healthy donors and NPC patients (Additional file 5: Figure S4).

To determine whether neuronal cells obtained from NPC patients retained the characteristic NPC phenotype, intracellular accumulation of cholesterol and gangliosides was analyzed by filipin staining and immunofluorescence, respectively. As shown in Figure 3, a massive accumulation of unesterified cholesterol was found in the bodies of differentiated cells obtained from NPC patients (Figure 3B). Furthermore, a percentage of these cells accumulated GM2 ganglioside as well (Figure 3D). Interestingly, the percentage of GM2 positive cells obtained applying the neuronal differentiation protocol to hSKIN-MASC from either NPC patients or a patient affected by Sandhoff disease was comparable (Figure 3F). GM2 accumulation was acquired during the differentiation process since this ganglioside could not be detected both in NPC fibroblasts (data not shown) and in undifferentiated hSKIN-MASC (Figure 3C). No accumulation of GM3 was detected both in normal and NPC cells (data not shown).

\section{Morphologic analysis of neuronal cells}

As mentioned above, NPC differentiated cells presented a morphology that was clearly different from normal cells. Therefore, in order to quantify the morphologic differences observed, we analyzed the neurite maximal and average length, neurite extremity, segment and root count and neurite node points, using the Neurite outgrowth image program on cells that stained positive for MAP-2. Statistically significant differences were found for all the analyzed parameters between normal and NPC neuronal cell. As shown in Figure 4, NPC differentiated cells presented longer neurites and a greater number of neurite extremities, segments, roots and node points.

\section{Discussion}

The remarkable advances in the ability to obtain stem cells from different tissues, including skin (reviewed in $[13,14]$ and [23]), which are able to differentiate into several cell lineages, offer the possibility to generate cells with neuronal characteristics from easily accessible sources.

It is conceivable then to develop human cellular models for the study of neurodegenerative diseases harvesting cells from skin biopsies of affected patients and committing them to a neuronal fate, thus paving the way to the study of pathways involved in the development of neurological diseases at the cellular level. 

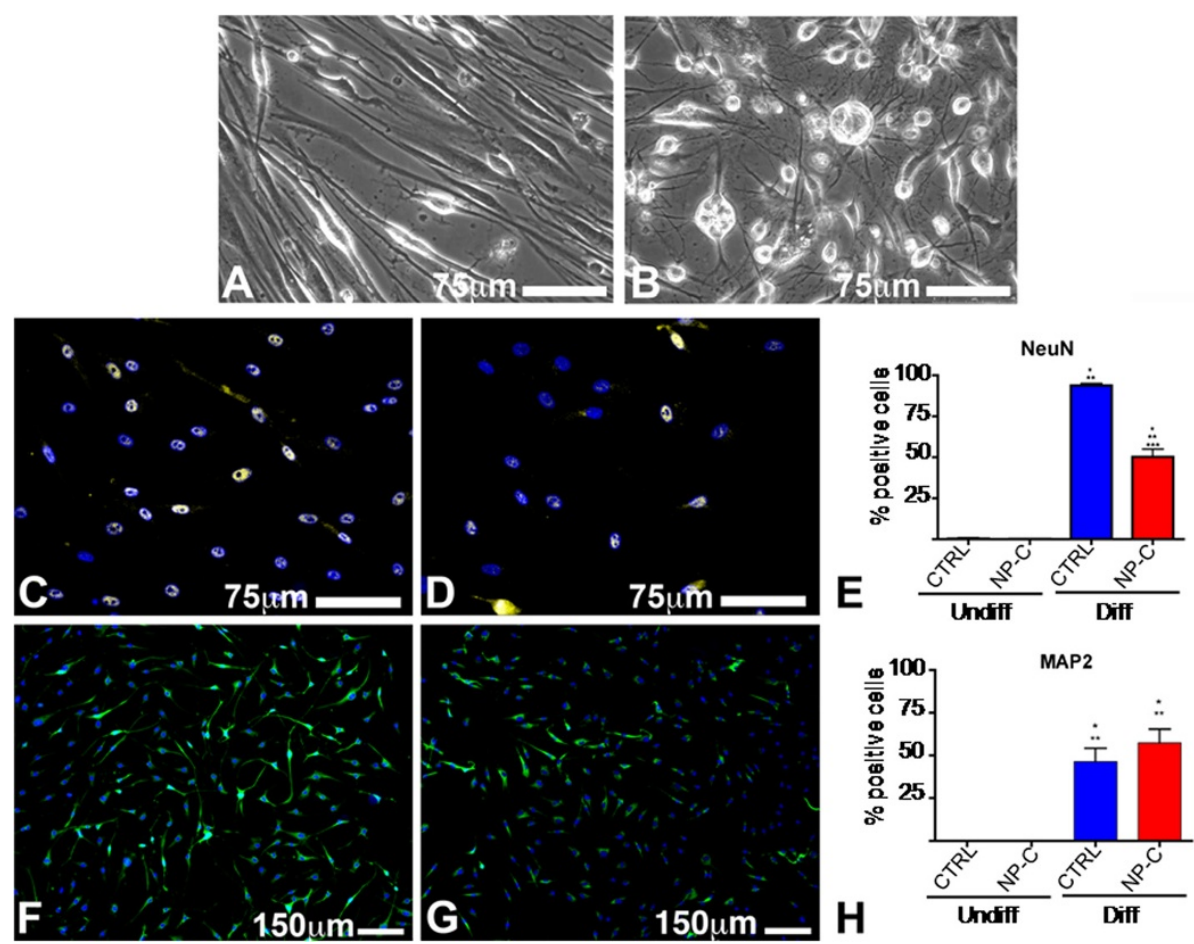

Figure 2 Neural differentiation of hSKIN-MASC obtained from already established skin fibroblast cultures. (A-B) Phase contrast images of hSKIN-MASC after induced to differentiate toward a neuronal fate (5 days in N2 medium $+48 \mathrm{~h}$ in N3 medium, see methods) from a healthy donor (A) and a NPC patient (B). (C-H) Neuronal markers detection: differentiated cells (5 days in N2 medium $+48 \mathrm{~h}$ in N3 medium, see methods), obtained from a healthy donor $(\mathbf{C}, \mathbf{F})$ or a NPC patient $(\mathbf{D}, \mathbf{G})$, express the neuron specific markers NeuN (yellow fluorescence, $\mathbf{C}, \mathbf{D})$ and MAP2 (green fluorescence, $\mathbf{F}, \mathbf{G}$ ). Nuclei are depicted by the blue fluorescence of DAPI staining. Quantitative evaluation of the percentage of cells expressing NeuN (E) or MAP2 $(\mathbf{H})$ in cultures from healthy donors $(C T R L, n=3)$ and NPC patients $(n=3)$ skin fibroblast derived cultures, before (Undiff.) and after the exposure to neuron induction media (Diff.). At least 400 cells have been counted for each cell line. Data are presented as mean \pm SD of 3 independent experiments one-way Anova test followed by Bonferroni post-test were utilized to compare means between groups. $P$ values less than 0.05 were considered significant. P values less than 0.05 were considered significant. *, **, ***, $p<0.05$ vs. columns 1,2 , and 3, respectively.

NPC is a genetic severe neurodegenerative disease characterized by the accumulation of cholesterol and other lipids within the late endosomes and lysosomes. Although the neuronal degeneration is the main feature in NPC patients, the molecular pathways linking the lipid accumulation and cellular damage in the central nervous system are largely unknown.

Therefore, we developed a human neuronal model of NPC disease using a method based on the induction of neuronal differentiation of stem cells isolated from skin biopsies or primary cultures of fibroblasts obtained from NPC patients.

Here, we demonstrated that it is possible to isolate clonogenic adult stem cells expressing mesenchymal and pluripotent state associated markers both from skin biopsies and fibroblast cultures. In analogy with the multipotent adult stem cells that we have previously isolated from several adult human tissues, these cells were widely multipotent, being able to differentiate into derivatives of the three germ layers [19,20]. For these reasons we named them hSKIN-MASC. The fact that we could obtain multipotent stem cells only from early passages of fibroblasts suggests that immature progenitors may be retained in those cultures but are lost upon serial expansion in vitro.

In particular, cells differentiated to neuronal fate expressed markers of mature neurons (NeuN and MAP2) and displayed morphological features resembling neuronal cells. It is worth to highlight that nestin, a marker of the early ectoderm lineage, was expressed both in neural stem cells and in hSKIN-MASC. These data suggest that hSKIN-MASC may represent a population of cells that share some features with the ectoderm lineage. This observation also suggests a common origin between hSKINMASC and Skin-derived Precursors or SKP, a multipotent cell population isolated from human dermis that shows a differentiation potential towards both mesenchymal and neural lineages. SKP are believed to be of neural-crest origin [24] and are retained throughout adulthood, although they decrease with age [25].

A preliminary characterization of the type of neuronal cells generated by this method, suggests that under the experimental conditions described, hSKIN-MASCs differentiated to cholinergic but not to dopaminergic or GABAergic neurons. 


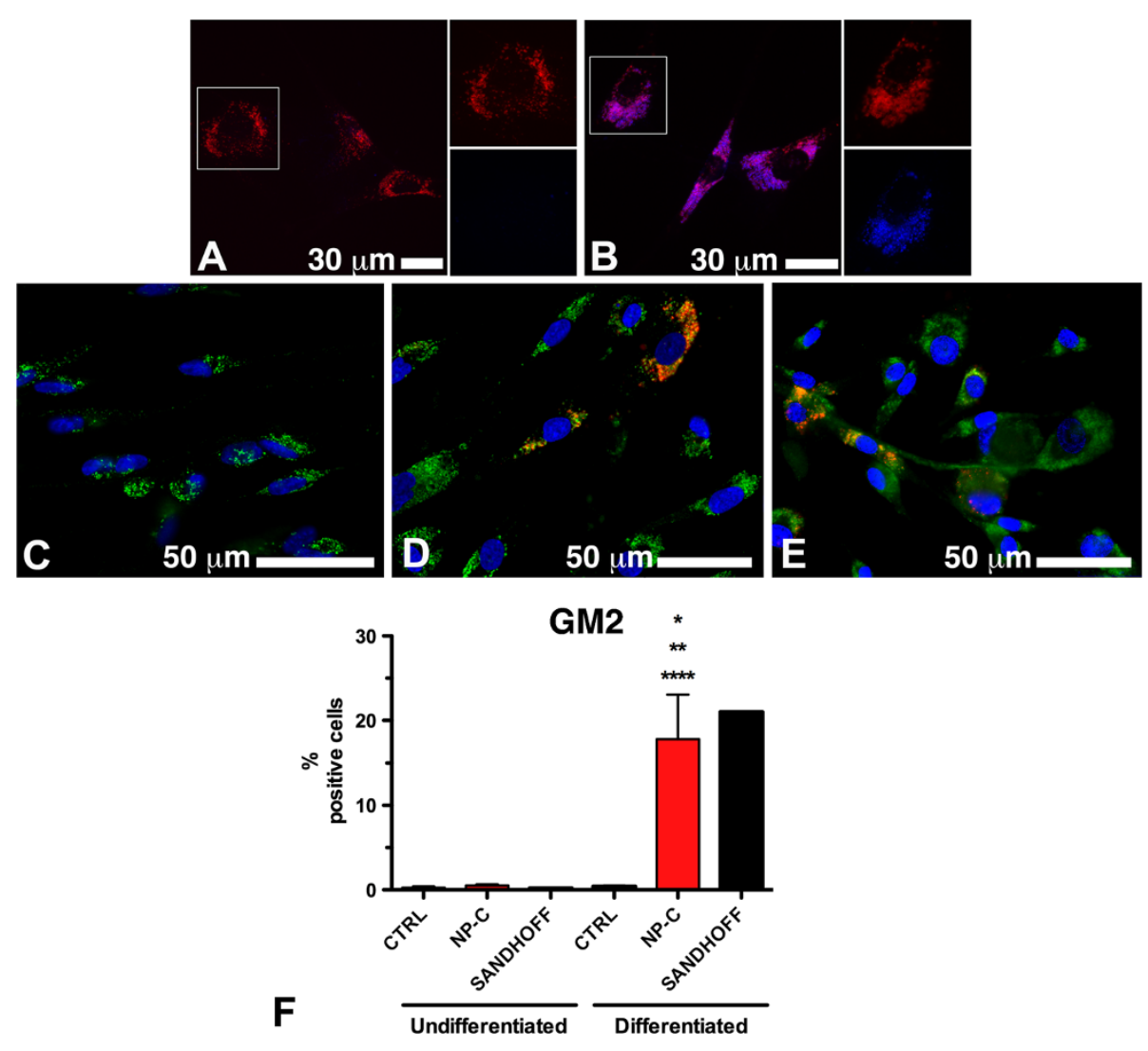

Figure 3 Pathologic accumulation of unesterified cholesterol and GM2 in differentiated hSKIN-MASC derived from NPC patients. (A -B) co-immunostaining for the lysosome marker Limp 1 (red fluorescence) and for unesterified cholesterol (blue fluorescence), revealed lysosomal accumulation of cholesterol after neuronal differentiation (5 days in N2 medium $+48 \mathrm{~h}$ in N3 medium, see methods) only in cultures derived from an NPC patient (B), but not in healthy donor derived cells (A). (C-F) Expression and relative quantification of the glycosphingolipid GM2 by immunofluorescence: co-immunostaining for the lysosome marker Limp 1 (green fluorescence) and for GM2 (red fluorescence), revealed accumulation of the ganglioside only in differentiated cultures derived from NPC (D) and Sandhoff (E) patients, but not in healthy donor derived cells (C). F) The percentage of GM2 expressing cells was assayed in undifferentiated cells and after exposure to the neural inductive media ( 5 days in N2 medium $+48 \mathrm{~h}$ in N3 medium, see methods), and compared to cells obtained in the same way but from a patient affected by Sandhoff disease, a specific GM2 gangliosidosis. No GM2 accumulation was detected in both healthy controls $(C T R L, n=3)$ and NPC $(n=3)$ undifferentiated cells, whereas a significant fraction of differentiated NPC $(n=3)$ and GM2 gangliosidosis derived cells showed accumulation of the glycosfingolipid. At least 400 cells have been counted for each cell line. Data are presented as mean \pm SD of 3 independent experiments; one-way Anova test followed by Bonferroni post-test were utilized to compare means between groups. P values less than 0.05 were considered significant. * **,***, p<0.05 vs. columns 1, 2 and 4, respectively. The Sandhoff patient was omitted from the statistical analysis.

Differentiated cells obtained from NPC patients showed some characteristic features of NPC phenotype. They accumulate unesterified cholesterol and GM2 ganglioside. The accumulation of GM2 ganglioside observed in the differentiated cells but not in fibroblasts or undifferentiated hSKIN-MASC, is consistent with data previously reported in NPC patients and NPC mouse models [10,12,26-29]. However, while these studies reported that NPC neuronal cells also accumulate relevant amounts of GM3 ganglioside, no significant accumulation of this ganglioside was found in our model. Since it has been described that the accumulation of GM2 in the brain precedes the accumulation of GM3 [10], it is possible to hypothesize that the model developed here recapitulates the early stage of the disease.
Furthermore, differentiated cells obtained from NPC patients displayed morphological features that are clearly different from those observed in cells obtained from healthy donors, such as the presence of longer neurites and a greater number of neurite extremities, segments, roots and node points. Interestingly, a distortion of the neuronal shape and an extensive growth of new ectopic neurites have been observed in cortical neurons obtained from human patients or animal models of NPC $[29,30]$. In addition, although there is no clear loss of cholinergic neurons in NPC mice, it has been shown that in these animals cholinergic neurons display several morphological alterations [31].

The identification of morphological alterations in differentiated NPC cells that seem to recapitulate what has 

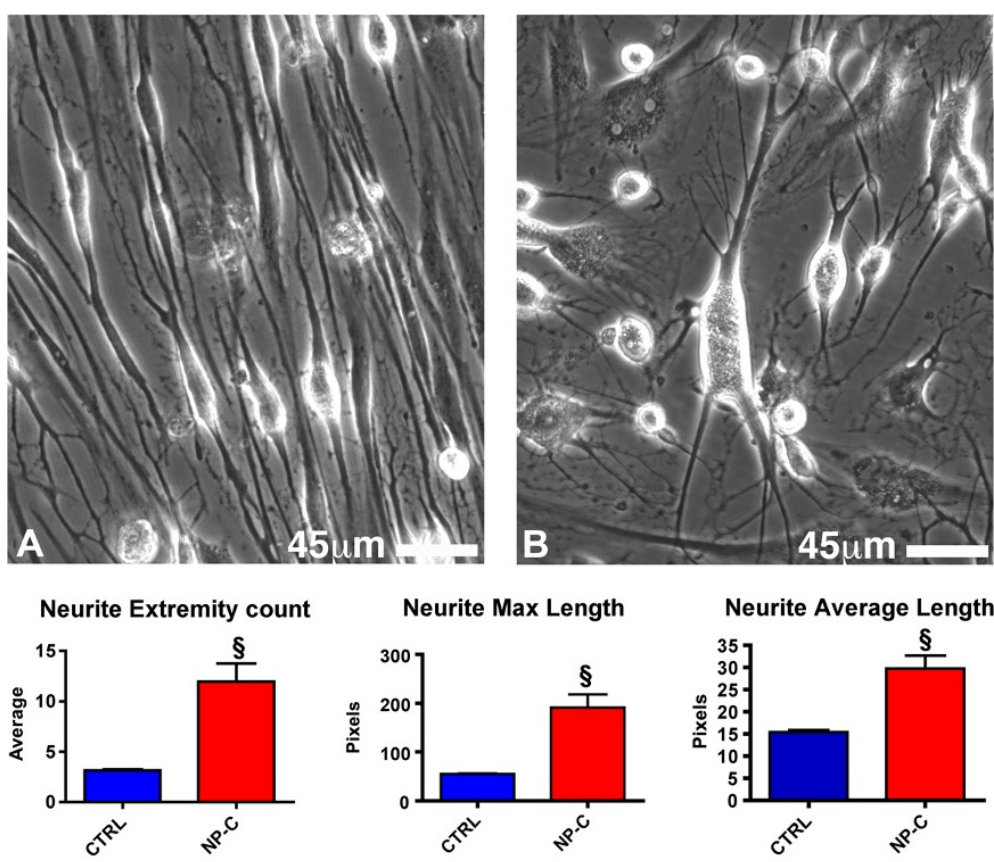

Neurite Node Points
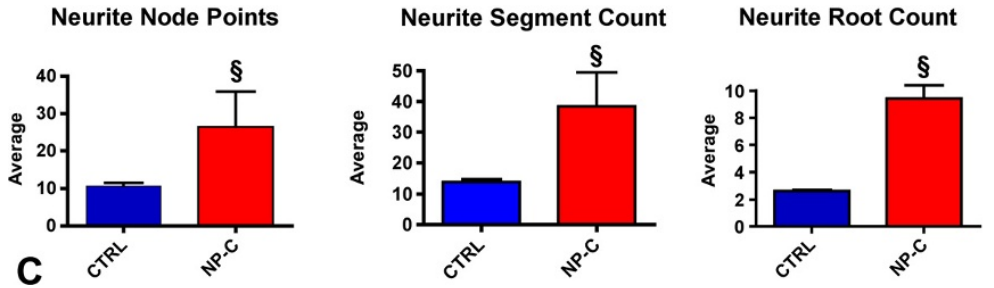

Figure 4 Morphometric analysis of differentiated cells obtained from hSKIN-MASC of healthy donors or NPC patients. (A- B): Phase contrast image showing the morphological differences between differentiated cells (5 days in N2 medium +48 h in N3 medium, see methods) of a healthy donor (A) and a NPC patient (B). Specifically, NPC differentiated cells were characterized by distortion of neuron shape, ectopic neurites and meganeurite formation. (C): Quantitative analysis of neuronal morphological parameters linked to neurite outgrowth as evaluated by a specific Image analysis software on MAP-2 stained cells. Data are presented as mean \pm SD of 3 independent experiments; $\S, p<0.01$ vs healthy donors (CTRL).

been described in human cortical NPC neurons is certainly fascinating. However, we cannot exclude that this phenotype is secondary to an impaired/altered differentiation of MASC isolated from NPC patients. Indeed, the lower percentage of cells expressing NeuN obtained after differentiation of hSKIN-MASC isolated from NPC patients seems to support this hypothesis. Further studies are planned to dissect the molecular mechanisms involved in this phenomenon.

Recently, two human cellular models of NPC disease have been developed by the specific silencing of NPC1 gene in the SH-SY5Y cells line [32] or in human embryonic stem cells [33]. Both models recapitulate the NPC biochemical phenotype. Although neuronal models obtained through the down-regulation of NPC1 expression in human cells may be useful to analyze some aspects of NPC pathogenesis, they are not suitable for the analysis of the impact of specific mutations on the pathologic phenotype or for testing mutation specific therapeutic strategies.
The model described here offers three main advantages with respect to the models cited above: 1 -it was developed directly from patient's cells and therefore it would be useful to analyze the effect of specific NPC1 mutations within the context of the patient genetic/epigenetic background; 2-it was obtained through the differentiation of cells obtained from accessible sources, such as patients cultured fibroblasts, usually available in many laboratories for diagnostic purposes; 3-it did not involve the forced expression of transgenes in target cells, thus avoiding confounding results due to the reprogramming process.

\section{Conclusion}

We have demonstrated that it is possible to isolate stem cells from skin biopsies or fibroblasts in culture and to commit them to a neuronal lineage. Differentiated cells obtained from NPC patients present the main features of NPC disease. Therefore, this model will be useful to study the molecular basis of NPC neurodegeneration 
and might represent a powerful tool to perform drug screening on cells obtained from NPC patients presenting different genotypes. In addition, the strategy described here may be applied to easily generate human neuronal models of other neurodegenerative diseases.

\section{Additional files}

Additional file 1: Table S1. Sequences of oligonucleotides used for real-time PCR.

Additional file 2: Figure S1. Mesodermic differentiation of hSKIN-MASC obtained from already established skin fibroblast cultures (hSKIN-MASC). (A-B) Phase contrast images of healthy donor- (A) and NPC patient- (B) derived hSKIN-MASC after exposure to a medium added with IGF-1, bFGF and VEGF. (C-F) Myocyte marker detection: differentiated cells, obtained from healthy donor $(C, E)$ or NPC patient $(D, F)$, express the myocyte specific markers alphasarcomeric actin (ASA) (red fluorescence, C,D) and smooth muscle actin (SMA) (red fluorescence, E,F). (G-H) Endothelial cell marker detection: differentiated cells, obtained from healthy donor $(\mathrm{G})$ or NPC patient $(\mathrm{H})$ express CD31 (red fluorescence, $\mathrm{G}, \mathrm{H}$ ). Nuclei are depicted by the blue fluorescence of DAPI staining. (I) Quantitative evaluation of the percentage of cells expressing ASA (i), SMA(ii) and CD31(iii) in cultures from healthy donors ( $C T R L, n=3)$ and NPC patients $(n=3)$, before (Undiff.) and after exposure to myocyte differentiation induction media (Diff.). At least 400 cells have been counted for each cell line. Data are presented as mean \pm SD; one-way Anova test followed by Bonferroni post-test was utilized to compare means between groups. ${ }^{*},{ }^{* *},{ }^{* *}, p<0.05$ vs columns 1,2 and 3 , respectively.

Additional file 3: Figure S2. Hepatic differentiation of hSKIN-MASC obtained from already established skin fibroblast cultures. (A-B) Phase contrast images of healthy donor- (A) and NPC patient- (B) derived hSKINMASC after differentiation into hepatocytes. (C-F) Hepatic markers detection: differentiated cells, obtained from healthy donor (C,E) or NPC patient $(D, F)$ express the hepatocytes specific markers cytokeratins 8-18-19 (red fluorescence, C,D) and stained positive for the Periodic Acid-Shiff (PAS) staining (pink stain, E,F). Nuclei are depicted by the blue fluorescence of DAPI staining ( $C, D)$ or by the blue-stain of hematoxylin (E, F). (G) Quantitative evaluation of the percentage of cells expressing CK in cultures from healthy donors (CTRL, $n=3$ ) and NPC patients $(n=3)$, before (Undiff.) and after exposure to hepatocytes differentiation induction media (Diff.). At least 400 cells have been counted for each cell line. Data are presented as mean $\pm S D$; one-way Anova test followed by Bonferroni post-test were utilized to compare means between groups. P values less than 0.05 were considered significant. ${ }^{*}{ }^{* *}, p<0.05$ vs column 1 and 2 , respectively.

Additional file 4: Figure S3. Relative expression of CHAT mRNA in cells derived from healthy donors and NPC patients. The relative abundance of CHAT mRNA were analyzed by real time PCR in cultures from healthy donors $(C T R L, n=3)$ and NPC patients ( $n=3$ ), before (Undiff.) and after neuronal. differentiation (Diff.; 5 days in N2 medium +48 h in N3 medium, see methods). Data were normalized by the expression of GAPDH and expressed as mean as mean \pm SD of 3 independent experiments.

Additional file 5: Figure S4. Apoptosis in differentiated hSKIN-MASC derived from healthy donors and NPC patients. After induction of neural differentiation ( 5 days in N2 medium $+48 \mathrm{~h}$ in N3 medium, see methods) the levels of apoptosis were evaluated in cultures derived from healthy donors (CTRL, $n=3)$ and NPC patients $(n=3)$. Data are presented as mean \pm SD of 3 independent experiments.

\section{Abbreviations}

NPC: Niemann Pick C; MASCs: Multipotent adult stem cells; FBS: Fetal bovine serum; DMEM: Dolbecco's Modified Eagle Medium; BSA: Albumin from bovine serum; PDGF: Platelet derived growth factor; EGF: Epidermal growth factor; bFGF: Basic fibroblast growth factor; VEGF: Vascular endothelial growth factor; IGF1: Insulin-like growth factor 1; HGF: Hepatocyte growth factor; NDS: Normal donkey serum; PAS: Periodic acid Schiff staining; CD31: Platelet endothelial cell adhesion molecule; SMA: Smooth muscle actin; ASA: a-sarcomeric actin; CK: Cytokeratins; MAP2: Microtubuleassociated protein 2; GFAP: Glial fibrillary acidic protein; O4: Oligodendroglial protein 4; TH: Tyrosine hydroxylase; DAT: Dopamine transporter; CHAT: Choline acetyltransferase; GAD: Glutamic acid decarboxylase; hMASC: Human multipotent adult stem cells; hSKIN-MASC: Human multipotent adult stem cells derived from skin biopsies and fibroblast cultures; SKP: Skin-derived Precursors.

\section{Competing interest}

The authors declare that they have no competing interests.

\section{Authors' contribution}

NB: performed the experiments and the statistical analysis. AD: conceived the idea and drafted the manuscript. A B: designed the experiments and helped to draft the manuscript. DC: designed the experiments and helped to draft the manuscript. SR: performed the experiments. SZ: performed the cholesterol and glycolipid analysis. RD: performed the single cell and multipotency experiments. BB: Provide clinical assistance and helped to draft the manuscript. CAB: Coordinate and directed the study. All authors read and approved the final manuscript.

\section{Acknowledgments}

The authors would like to thank Dr. Konstantin Dobrenis for providing antiGM2 and anti-GM3 antibodies. This work was supported by the Italian Association of Niemann Pick,a grant from the Italian Health Ministry "Role of protein misfolding in the pathogenesis of Niemann-Pick type C disease:a possible therapeutic target (RF-2009-1524462)" and a grant from Italian Health Ministry GR-2007-683407.

\section{Author details}

${ }^{1}$ Department of Medical and Biological Sciences (DSMB), University of Udine, P.le Kolbe 3, 33100, Udine, Italy. ${ }^{2}$ Regional Coordinator Centre for Rare Diseases, University Hospital "Santa Maria della Misericordia", Udine, Italy.

Received: 7 September 2012 Accepted: 14 February 2013

Published: 21 February 2013

\section{References}

1. Patterson M, Vanier MT, Suzuki K, Morris ED, Cartsea EB, Neufeld EJ, Blanchette-Mackie PG, Pentchev: Niemann Pick disease type C: a lipid trafficking disorder. In The metabolic and molecular basis of inherited diseases, Volume 3. VIlth edition. Edited by Scriver CR, Beaudet AL, Sly WS, Valle D. New York: Mc Graw-Hill; 2001:611-634.

2. Vanier MT, Millat G: Niemann-Pick disease type C. Clin Genet 2003, 64:269-281

3. Carstea ED, Polymeropoulos MH, Parker CC, Detera-Wadleigh SD, O'Neill RR, Patterson MC, Goldin E, Xiao H, Straub RE, Vanier MT, Roscoe OB, Pentchev PG: Linkage of Niemann-Pick disease type $C$ to human chromosome 18. Proc Natl Acad Sci U S A 1993, 90:2002-2004

4. Vanier MT, Duthel S, Rodriguez-Lafrasse C, Pentchev P, Carstea ED: Genetic heterogeneity in Niemann-Pick $C$ disease: a study using somatic cell hybridization and linkage analysis. Am J Hum Genet 1996, 58:118-125.

5. Davies JP, loannou YA: Topological analysis of Niemann-Pick C1 protein reveals that the membrane orientation of the putative sterol-sensing domain is identical to those of 3-hydroxy-3-methylglutaryl-CoA reductase and sterol regulatory element binding protein cleavage-activating protein. J Biol Chem 2000, 275:24367-24374

6. Naureckiene S, Sleat DE, Lackland H, Fensom A, Vanier MT, Wattiaux R, Jadot M, Lobel P: Identification of HE1 as the second gene of Niemann-Pick C disease. Science 2000, 290:2298-2301.

7. Boothe AD, Kruth HS, Weintroub H, Stivers J, Brady RO: A genetic storage disorder in BALB/C mice with a metabolic block in esterification of exogenous cholesterol. J Biol Chem 1984, 259:5784-5791.

8. Maue RA, Burgess RW, Wang B, Wooley CM, Seburn KL, Vanier MT, Rogers MA, Chang CC, Chang TY, Harris BT, Graber DJ, Penatti CA, Porter DM, Szwergold BS, Henderson LP, Totenhagen JW, Trouard TP, Borbon IA, Erickson RP: A novel mouse model of Niemann-Pick type C disease carrying a D1005G-Npc1 mutation comparable to commonly observed human mutations. Hum Mol Genet 2012, 21:730-750.

9. Love S, Bridges LR, Case CP: Neurofibrillary tangles in Niemann-Pick disease type C. Brain 1995, 118:119-129.

10. Walkley SU, Suzuki K: Consequences of NPC1 and NPC2 loss of function in mammalian neurons. Biochim Biophys Acta 2004, 1685:48-62. 
11. Vance JE, Karten B, Hayashi H: Lipid dynamics in neurons. Biochem SoC Trans 2006, 34:399-403.

12. Zervas M, Somers KL, Thrall MA, Walkley SU: Critical role for glycosphingolipids in Niemann-Pick disease type C. Curr Biol 2001, 11:1283-1287

13. Beltrami AP, Cesselli D, Beltrami CA: Pluripotency rush! Molecular cues for pluripotency, genetic reprogramming of adult stem cells, and widely multipotent adult cells. Pharmacol Ther 2009, 124:23-30.

14. Ratajczak MZ, Zuba-Surma E, Kucia M, Poniewierska A, Suszynska M, Ratajczak J: Pluripotent and multipotent stem cells in adult tissues. Adv Med Sci 2012, 57:1-17.

15. Kern S, Eichler $H$, Stoeve J, Klüter $H$, Bieback K: Comparative analysis of mesenchymal stem cells from bone marrow, umbilical cord blood, or adipose tissue. Stem Cells 2006, 24:1294-1301.

16. Anghileri S, Marconi S, Pignatelli A, Cifelli P, Galié M, Sbarbati A, Krampera M, Belluzzi O, Bonetti B: Neuronal differentiation potential of human adiposederived mesenchymal stem cells. Stem Cells Dev 2008, 17:909-916.

17. Seo MJ, Suh SY, Bae YC, Jung JS: Differentiation of human adipose stromal cells into hepatic lineage in vitro and in vivo. Biochem Biophys Res Commun 2005, 328:258-264.

18. Case J, Horvath $\mathrm{TL}$, Ballas CB, March $\mathrm{KL}$, Srour EF: In vitro clonal analysis of murine pluripotent stem cells isolated from skeletal muscle and adipose stromal cells. Exp Hematol 2008, 36:224-234.

19. Beltrami AP, Cesselli D, Bergamin N, Marcon P, Rigo S, Puppato E, D'Aurizio F, Verardo R, Piazza S, Pignatelli A, Poz A, Baccarani U, Damiani D, Fanin R, Mariuzzi L, Finato N, Masolini P, Burelli S, Belluzzi O, Schneider C, Beltrami CA: Multipotent cells can be generated in vitro from several adult human organs (heart, liver and bone marrow). Blood 2007, 110:3438-3446.

20. Cesselli D, Beltrami AP, Rigo S, Bergamin N, D'Aurizio F, Verardo R, Piazza S, Klaric E, Fanin R, Toffoletto B, Marzinotto S, Mariuzzi L, Finato N, Pandolfi M, Leri A, Schneider C, Beltrami CA, Anversa P: Multipotent progenitor cells are present in human peripheral blood. Circ Res 2009, 104:1225-1234.

21. Blanchette-Mackie EJ, Dwyer NK, Amende LM, Kruth HS, Butler JD, Sokol J, Comly ME, Vanier MT, August JT, Brady RO: Type-C Niemann-Pick disease: low density lipoprotein uptake is associated with premature cholesterol accumulation in the Golgi complex and excessive cholesterol storage in lysosomes. Proc Natl Acad Sci U S A 1988, 85:8022-8026.

22. Zhou S, Davidson C, McGlynn R, Stephney G, Dobrenis K, Vanier MT, Walkley SU: Endosomal/lysosomal processing of gangliosides affects neuronal cholesterol sequestration in Niemann-Pick disease type C. Am J Pathol 2011, 179:890-902.

23. Hunt DP, Jahoda C, Chandran S: Multipotent skin-derived precursors: from biology to clinical translation. Curr Opin Biotechnol 2009, 20:522-530.

24. Fernandes KJ, McKenzie IA, Mill P, Smith KM, Akhavan M, Barnabé-Heider F, Biernaskie J, Junek A, Kobayashi NR, Toma JG, Kaplan DR, Labosky PA, Rafuse $V$, Hui CC, Miller FD: A dermal niche for multipotent adult skinderived precursor cells. Nat Cell Biol 2004, 6:1082-1093.

25. Gago N, Perez-Lopez V, Sanz-Jaka JP, Cormenzana P, Eizaguirre I, Bernad A, Izeta A: Age-dependent depletion of human skin-derived progenitor cells. Stem Cells 2009, 27:1164-1172.

26. Elleder M, Jirasek A, Smid F, Ledvinova J, Besley GTN: Niemann-Pick disease Type C: study on the nature of the cerebral storage process. Acta Neuropathol (Berl) 1985, 66:325-336.

27. Vanier MT: Lipid changes in Niemann-Pick disease type $C$ brain: personal experience and review of the literature. Neurochem Res 1999, 24:481-489.

28. Sleat DE, Wiseman JA, El-Banna M, Price SM, Verot L, Shen MM, Tint GS, Vanier MT, Walkley SU, Lobel P: Genetic evidence for nonredundant functional cooperativity between NPC1 and NPC2 in lipid transport. Proc Natl Acad Sci USA 2004, 101:5886-5891.

29. March PA, Thrall MA, Brown DE, Mitchell TW, Lowenthal AC, Walkley SU: GABAergic neuroaxonal dystrophy and other cytopathological alterations in feline Niemann-Pick disease type C. Acta Neuropathol 1997, 94:164-172.

30. Zervas M, Dobrenis K, Walkley SU: Neurons in Niemann-Pick disease type $\mathrm{C}$ accumulate gangliosides as well as unesterified cholesterol and undergo dendritic and axonal alterations. J Neuropathol Exp Neurol 2001, 60:49-64.

31. German DC, Quintero EM, Liang CL, Ng B, Punia S, Xie C, Dietschy JM: Selectiveneurodegeneration, without neurofibrillary tangles, in a mouse model of Niemann-Pick C disease. J Comp Neurol. 2001, 433:415-425.
32. Rodríguez-Pascau L, Coll MJ, Casas J, Vilageliu L, Grinberg D: Generation of a human neuronal stable cell model for Niemann-pick $C$ disease by RNA interference. JIMD Reports 2012, 4:29-37.

33. Ordonez MP, Roberts EA, Kidwell C, Yuan S, Plaisted W, Goldstein LS: Disruption and therapeutic rescue of autophagy in a human neuronal model of Niemann Pick type C1. Hum Mol Genet 2012, 21:2651-2662.

doi:10.1186/1750-1172-8-34

Cite this article as: Bergamin et al:: A human neuronal model of Niemann Pick $C$ disease developed from stem cells isolated from patient's skin. Orphanet Journal of Rare Diseases 2013 8:34.

\section{Submit your next manuscript to BioMed Central and take full advantage of:}

- Convenient online submission

- Thorough peer review

- No space constraints or color figure charges

- Immediate publication on acceptance

- Inclusion in PubMed, CAS, Scopus and Google Scholar

- Research which is freely available for redistribution 